Am J Psychiatry Vol. 130(2):165-170 (1973)

ISSN: (print 0002-953X)(online 1535-7228)

This is a peer reviewed pre-print version of the following article: Levoamphetamine and Dextroamphetamine:

Differential Effect on Aggression and Hyperkinesis in Children and Dogs, which has been published in final form at:

http://www.appi.org

http://www.appi.org/SearchCenter/Pages/Journal.aspx?ItemId=AJP

http://ajp.psychiatryonline.org/article.aspx?articleid=153038

(C) 1973 American Psychiatric Publishing, Inc.

\title{
Levoamphetamine and Dextroamphetamine: Differential Effect on Aggression and Hyperkinesis in Children and Dogs
}

\author{
By L. Eugene Arnold, M.D., Vladimir Kirilcuk, M.D., PH.D., \\ Samuel A. Corson, PH. D., and Elizabeth O'L. Corson, M.S.
}

\begin{abstract}
In laboratory experiments with hyperkinetic, untrainable dogs and in a comparison of levoamphetamine, dextroamphetamine, and placebo in children, levoamphetamine and dextroamphetamine were found to be approximately equal in calming an aggressive, hostile dog and in benefiting "unsocialized-aggressive" children; dextroamphetamine was more effective than levoamphetamine in calming "nervousness" and hyperactivity in dogs and in overanxious-hyperkinetic children. These data suggest that in the hyperkinetic syndrome, aggression and hostility may be benefited equally by levoamphetamine or dextroamphetamine via a dopaminergic mechanism, while anxiety and overactivity may be benefited significantly only by the dextro isomer via a norepinephrinergic mechanism.
\end{abstract}

The plight of children with behavior and learning problems that often respond to stimulants by "paradoxical" calming has become well known under such names as "hyperkinetic syndrome," "minimal brain dysfunction," and "minimal brain damage." These and other grammatically singular appellations are bandied as if we believed these problems represented a single, homogeneous clinical entity (like pneumococcal pneumonia) that would uniformly respond to a prescribed treatment, if only we could find the treatment. However, those familiar with the syndrome readily admit its diversity of clinical pictures, probable diversity of etiology and pathogenesis, and even diversity of response to the highly touted stimulant therapy.

At least two authors have recently attempted to clean up this diagnostic cesspool. Fish (1) has proposed criteria by which children who may respond favorably to stimulants could be assigned to one of three diagnostic categories: 308.0-hyperkinetic reaction (with immaturity, inadequacy, lability, and poor organization); 308.2- overanxious reaction ("nervousness" and possible overactivity with subjective distress, otherwise well-organized behavior); and 308.4 - unsocialized-aggressive reaction (aggressiveness and hostility with denial of feelings and personal responsibility, otherwise well-patterned behavior). These categories are reminiscent of Wender's "classical hyperactive," "neurotic," and "sociopathic" subsyndromes (2).

One of the puzzling things about the hyperkinetic syndrome that raises serious questions about its being a homogeneous diagnostic entity is the individual variation in response to stimulants. It has long been known $(3,4)$ that sometimes dextroamphetamine helps a hyperkinetic child more than the racemic (dl) mixture does, and sometimes vice versa. In addition, some children are helped by methylphenidate (Ritalin) but not by amphetamine, and vice versa. Furthermore, while the majority of such children are helped by stimulants, a small percentage are actually made worse. The uncertainty concerning the practical clinical use of drugs for these children is underscored by recent attempts to introduce some prognostic guidelines. Barcai (5) has proposed a finger twitch test. Yoss and Moyer (6) have found that 25 percent of hyperkinetic and underachieving children have a narcoleptic-like electronic pupillogram that changes favorably with 
behavioral improvement on amphetamine. Knopp and associates (7) have found a correlation of approximately $0.6(\mathrm{p}<.01)$ between behavioral response to dextroamphetamine and changes in the extent of pupillary light reaction before and one-half hour after a test dose.

The question naturally arises whether the differential drug effects may be correlated with the different diagnostic categories proposed by Fish (1) and Wender (2). Clinical pharmacological separation of the aggressive and "nervous" components of the hyperkinetic syndrome seems to be supported by data from pilot psychopharmacologic experiments with hyperkinetic, untrainable dogs in the Laboratory of Cerebrovisceral Physiology of the Department of Psychiatry, Ohio State University in Columbus.

\begin{tabular}{lccc}
\hline Category & $\begin{array}{c}\text { Dextroampheta } \\
\text { mine vs. } \\
\text { Placebo }\end{array}$ & $\begin{array}{c}\text { Levoamphetamin } \\
\text { e vs. Placebo }\end{array}$ & $\begin{array}{c}\text { Dextroamphetamine vs. } \\
\text { Levoamphetamine }\end{array}$ \\
\hline $\begin{array}{l}\text { Psychiatrists' ratings } \\
\text { Unsocialized-aggressive group }\end{array}$ & $\mathrm{p}<.0001$ & $\mathrm{p}<.005$ & $\mathrm{n} . \mathrm{s}$. \\
Overanxious-hyperkinetic group & $\mathrm{p}<.03$ & $\mathrm{n} . \mathrm{s}$. & $\mathrm{p}<.005$ \\
$\begin{array}{l}\text { Teachers' ratings } \\
\text { Unsocialized-aggressive group }\end{array}$ & $\mathrm{p}<.05$ & $\mathrm{p}<.05$ & $\mathrm{n} . \mathrm{s}$. \\
$\quad$ Overanxious-hyperkinetic group & $\mathrm{p}<.05$ & n.s. & $\mathrm{p}<.05$ \\
$\begin{array}{l}\text { Parents' ratings } \\
\text { Unsocialized-aggressive group }\end{array}$ & $\mathrm{p}<.02$ & $\mathrm{p}<.02$ & n.s. \\
Overanxious-hyperkinetic group & n.s. & n.s. & n.s. \\
\hline
\end{tabular}

Table 1 Comparison of Significant Differences Between Treatments with Dextroamphetamine, Levoamphetamine, and Placebo

\section{Dog Experiments}

\section{Method and Results}

The four hyperkinetic, untrainable dogs used in this pilot study were previously reported on by Corson and associates $(8,9)$. Most of the dogs were merely hyperkinetic, not particularly vicious. One of them, however, a cocker-beagle, was both hyperkinetic and aggressively hostile, providing a neat animal model for the constellation of symptoms seen in some aggressive hyperkinetic children.

While aggressive behavior could be easily observed, even in the dog's home cage or kennel, the hyperkinetic traits could be best observed when a dog was placed on a Pavlovian stand, mildly restrained, and left alone in a soundproof chamber, as described by the Corsons elsewhere (10). Therefore, experiments were conducted in both settings.

In cage experiments the aggressive hyperkinetic dog was given either 1- or d-amphetamine (10 mg. orally) on different occasions. Each time, amphetamines had a very pronounced effect: By approximately one hour the ordinarily vicious dog completely lost his aggressiveness. It was possible to take him out of the cage, walk him, pet him, and feed him a treat without danger of the experimenter's being bitten.

Although both isomers were equally effective in suppressing aggression, the duration of effect was different: While d-amphetamine kept the dog quiet seven hours after administration, 1-amphetamine lasted only about half as long.

On the Pavlovian stand, "normal" dogs, after a few unsuccessful tries to escape, eventually give up fighting and tolerate the restraints. By contrast, "hyperkinetic" dogs immediately start thrashing, chewing equipment within reach, and trying to free themselves. This behavior would go 
on for indefinite numbers of experiments; they simply would not adapt to the experimental situation.

Pavlovian-stand experiments in which both isomers were used were carried out on four hyperkinetic dogs, including the vicious one. The dosages varied from 2.5 to $40 \mathrm{mg}$. (.25 to 4.0 mg./kg.). For each dog, elimination of all signs of hyperkinetic behavior required three to four times as much levoamphetamine as dextroamphetamine. The vicious dog, for example, required $40 \mathrm{mg}$. of levoamphetamine to eliminate hyperkinesis as effectively as $10 \mathrm{mg}$. of dextroamphetamine did, even though his aggression and hostility were eliminated by $10 \mathrm{mg}$. of either isomer.

This differential effect on hyperactivity paralleled the differential effect on appetite. The three dogs who showed anorexia did so with $1.0 \mathrm{mg} . / \mathrm{kg}$. of dextroamphetamine but required about three times that amount of levoamphetamine to produce anorexia. Unfortunately, dextroamphetamine had been tried before levoamphetamine in these exploratory experiments, so that tolerance cannot be ruled out as a source of the apparent difference in anorexogenic potency.

\section{Conclusions}

Thus, from pilot animal experiments in standardized laboratory settings, we have data suggesting that in hyperkinetic organisms: 1) levoamphetamine has a shorter duration of action than dextroamphetamine; 2) during the duration of maximal effect, levoamphetamine and dextroamphetamine seem to be approximately equal in their aggression-taming potency, but dextroamphetamine is three to four times as potent in calming "nervousness" and in producing anorexic effects; and 3) therefore, the beneficial effects of amphetamine on aggression and "nervousness" may operate through different biochemical mechanisms.

\section{Children}

\section{Method and Design}

Encouraged by these findings in laboratory dogs, we returned to the raw data of a previously reported nine-week double-blind crossover comparison of levoamphetamine, dextroamphetamine, and placebo (11) that demonstrated the efficacy of levoamphetamine in hyperkinetic children. This study had only 11 subjects, but it offered reliable, quantitative, independent ratings by psychiatrists, teachers, and parents. Without being consciously aware of how each child fared comparatively on the dextroamphetamine and levoamphetamine ratings, we assigned each of the children to one of the three diagnostic categories advocated by Fish (1). This resulted in five diagnoses of unsocialized-aggressive reaction (308.4), four diagnoses of overanxious reaction (308.2), and two diagnoses of hyperkinetic reaction (308.0). ${ }^{\mathrm{i}}$

Because these last two groups were so small and because we were mainly interested in aggression as opposed to other manifestations of minimal brain dysfunction, we lumped those with overanxious reaction and those with hyperkinetic reaction together as the "overanxious-hyperkinetic" group for statistical comparisons on the three sets of ratings.

\section{Results}

Figure 1 shows the psychiatrists' ratings for the two groups; they are mainly based on weekly parental quantification of target symptoms. The three independent raters had an overall interrater reliability coefficient of 0.7734 . Note that for the unsocialized-aggressive group levoamphetamine was rated as being almost as good as dextroamphetamine (difference not significant), and both of these active drugs were significantly better than placebo. In contrast, for the overanxious-hyperkinetic group the levoamphetamine was no better than placebo, even though 
dextroamphetamine was significantly better (see table 1).

The same pattern emerged when the two groups were compared on the teachers' behavior rating scale (figure 2). Here we find that in the unsocialized-aggressive group levoamphetamine was actually rated slightly better than dextroamphetamine (difference not significant). Both active drugs were significantly better than placebo. Again we find that in the overanxious-hyperkinetic group levoamphetamine is rated as not significantly better than placebo, and dextroamphetamine as significantly better than either placebo or levoamphetamine (table 1).

The parents' behavior checklist (figure 2) does not at first seem to show the same pattern. However, if we attend only to the differences that are statistically significant $(\mathrm{p}<.05$ level $)$, they do partially support the same pattern as the psychiatrists' and teachers' ratings. In the unsocialized-aggressive group both active drugs were rated as significantly better than placebo and not significantly different from each other. All differences for the overanxious-hyperkinetic group were not significant (see the results of comparison by one-tailed paired t test in table 1).

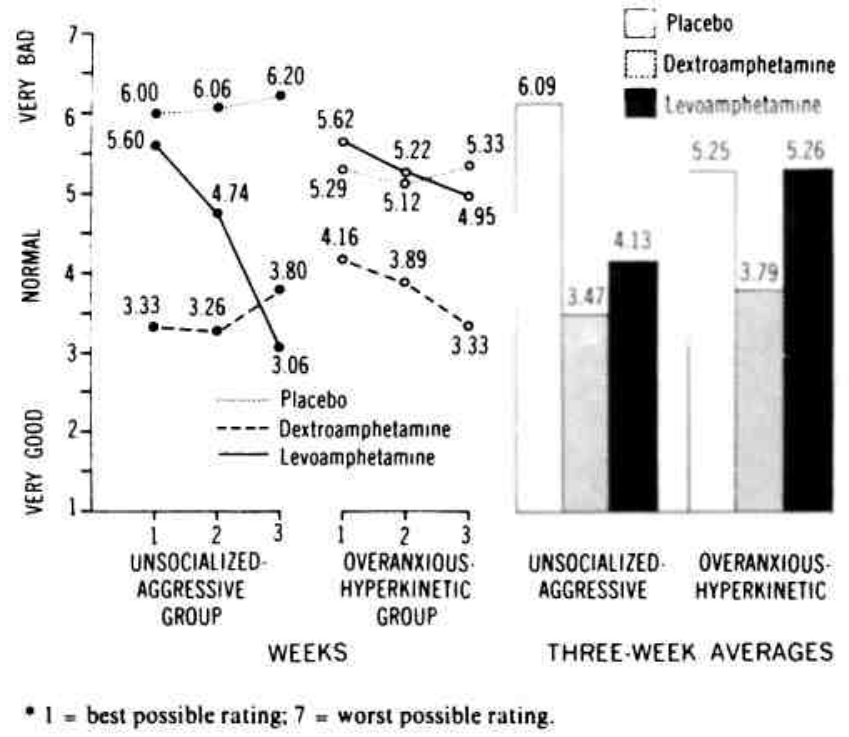

Fig. 1. Comparison of Two Diagnostic Groups on Psychiatrists' Ratings of Drug Effect* with Placebo, Dextroamphetamine, and Levoamphetamine

Individual factor scores for aggression, hyperactivity, and daydreaming-inattention on the pooled teachers' symptom ratings (11) likewise only partially showed the same pattern. The aggression factor showed comparable isomeric efficacy, as did the aggressive dog and the unsocialized-aggressive group. Further, the daydreaming-inattention factor showed a statistical gap in isomeric efficacy, as did the hyperkinetic dogs and the overanxious-hyperkinetic group.

However, the hyperactivity factor showed comparable efficacy, a flaw in the pattern seen in the rest of the data.

To rule out dosage variation as a source of the differences in efficacy, the average number of tablets given per day for each drug condition was tabulated by diagnosis (see table 2). The range of dosage is rather narrow, and what variation there is tends to dramatize the observed differences rather than question them. The clinicians had been instructed to optimize the dosage on each drug condition. Therefore an ineffective drug would tend to be given in higher dosages, as shown by the placebo doses. For the overanxious-hyperkinetic group, where levoamphetamine seemed ineffective, the drug was used in a dosage comparable to placebo, while dextroamphetamine, which was significantly more beneficial, was used in a much lower dose. 
For the unsocialized-aggressive group, where levoamphetamine seemed as beneficial as dextroamphetamine, levoamphetamine was used in lower doses.

\begin{tabular}{lcc}
\hline & \multicolumn{2}{c}{ Number of Tablets a Day } \\
\cline { 2 - 3 } Medication & $\begin{array}{c}\text { Unsocialized- } \\
\text { Aggressive Group }\end{array}$ & $\begin{array}{c}\text { Overanxious- } \\
\text { Hyperkinetic } \\
\text { Group }\end{array}$ \\
\hline Placebo & 6.9 & 7.0 \\
Dextroamphetamine* & 6.5 & 5.7 \\
Levoamphetamine* & 5.7 & 6.9 \\
\hline
\end{tabular}

$*$ One tablet $=5 \mathrm{mg}$. of amphetamine sulfate.

Table 2 Comparison of Two Diagnostic Groups on the Average Number of Tablets Used a Day for Each Drug Condition

\section{Discussion}

The teachers' ratings and the psychiatrists' ratings of weekly parental assessments of target symptoms in a double-blind crossover study on hyperkinetic children, as well as careful laboratory observations on a hyperkinetic aggressive dog, all suggest that aggressive, hostile behavior may be a distinct (if not separable) part of the hyperkinetic syndrome. It seems to be helped by levoamphetamine as much as by dextroamphetamine, in contrast to anxiety and overactivity, which appear to be helped significantly more by dextroamphetamine. The observations made on laboratory dogs demonstrate this contrast in the same organism; the data from the children's study show it from patient to patient. While the nonaggressive children who were merely hyperactive benefited significantly only from dextroamphetamine, the most aggressive children enjoyed approximately equal overall improvement when taking either levoamphetamine or dextroamphetamine.

With regard to the latter, levoamphetamine, insofar as it has fewer "central side effects" than dextroamphetamine, may eventually become the drug of choice for the aggressive, hostile subgroup of hyperkinetic children who would be diagnosed by Fish's criteria as having "unsocialized aggressive reaction" (308.4). However, this is far from established by the data presented here, especially in view of: 1 ) the small numbers of children in the clinical study, 2) the retrospective nature of the assignment to diagnostic categories, 3) the failure of parents' ratings and pooled teachers' rating factors to confirm all the findings on psychiatrists' ratings and teachers' rating totals, and 4) the preliminary nature of the dog experiments. Further investigations on both animals and children are obviously needed.

Whether levoamphetamine (and dextroamphetamine) may be useful in treating other kinds of aggression remains unclear at this point. Goldman, Lindner, and Dinitz at Ohio State University are initiating a double-blind comparison of placebo, levoamphetamine, dextroamphetamine, and imipramine in sociopathic convicts that may provide some information along this line. However, it may be argued that the aggression seen in sociopaths is not really a different kind; there is a suspicion that at least some sociopaths are hyperkinetic children grown up.

The differential response to levoamphetamine, if upheld by further investigations, would tend to validate Fish's proposed diagnostic groupings for children with behavior disorders. Here, for the first time, we may have the beginnings of a diagnosis-treatment situation analogous to diagnosing pneumococcal pneumonia and treating it with penicillin rather than diagnosing Hemophilus influenzae pneumonia and treating it with ampicillin. Obviously, there remain many unanswered questions, some of which follow. 
1. It would be difficult to guess from the currently available data whether or not the "classical hyperkinetic" subgroup of hyperkinetic children should be lumped together with the "overanxious" group with regard to drug response, whether it would be more like the "unsocialized-aggressive" group, or whether it would be different from either.

2. The place of learning disabilities in this scheme would need clarification. It is now known that some children who show primarily a learning disability without obvious behavior problems can also benefit from "stimulant" medication (12). Would such children in their drug response more closely parallel the unsocialized-aggressive group or the overanxious-hyperkinetic group, or would they constitute yet another group?

3. The possibility of synergism between the optical isomers of amphetamine remains unexplored. The biochemical literature scarcely recognizes the possibility except for isolated, brief speculation (13). In the clinical literature the occasional superiority of racemic (dl) amphetamine over pure dextroamphetamine is reported without explanation $(3,4)$. It could result from either synergism or merely an additive effect since the racemic mixture is usually given in higher doses than pure dextroamphetamine. Two clinical observations more strongly suggest a possible synergism:

A. Immediately upon completion of the double-blind, triple crossover comparison, six of the 11 children were given racemic amphetamine in the same total daily dose as an interim medication until the code was broken in order to determine which of the isomers they had fared best on. Out of curiosity, the clinician involved (L.E.A.) did not tell the teachers that the study had ended and had them fill out an additional rating scale for the child's behavior the first week he was on racemic amphetamine. Five of the parents also cooperated by filling out an additional parent's rating. This "piggyback" experiment was thus blind only in regard to the teachers' ratings. The racemic amphetamine was reported to be the most effective of all four drug conditions by both teachers' and parents' checklists for two of the children, by the teachers' checklist alone for one child, and by the parents' checklist alone for one child.

B. The other clinician in our original study (Keith R. McCloskey [11]) has since independently reported that he has found children who respond favorably to various ratios of dextro- and levoamphetamine but not to any other combination or to either isomer alone.

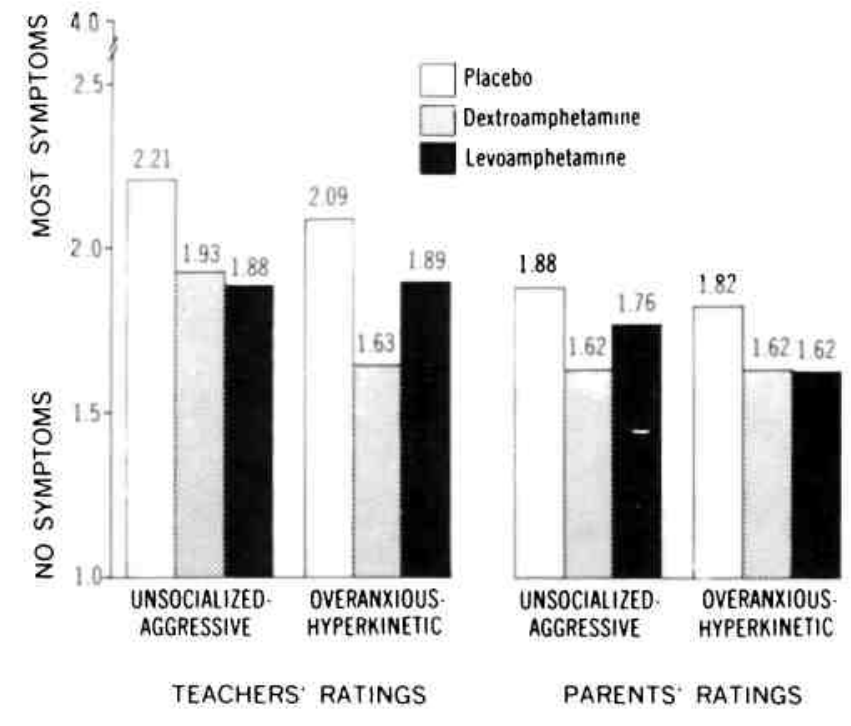

$\bullet 1=$ no symptoms; $4=$ most symptomatic. 
Fig. 2. Teachers' and Parents' Blind Ratings* of Two Diagnostic Groups on Placebo, Dextroamphetamine, and Levoamphetamine

Though both of these data are open to many criticisms, they do suggest that the potency relationships of the optical isomers of amphetamine may be more complex than has been previously realized and need to be further investigated both for their practical clinical applications and for their value in elucidating basic neurochemical phenomena.

In a previous paper (11), the reasons were detailed for suspecting that, insofar as the levo isomer's efficacy matches that of the dextro isomer, amphetamine may help hyperkinetic children via a dopaminergic mechanism, whereas a wide disparity between the efficacies of the two isomers would suggest a norepinephrinergic mechanism. Briefly, Snyder and associates (13) showed that dextroamphetamine is ten times as potent as levoamphetamine in inhibiting catecholamine uptake by norepinephrinergic terminals and in eliciting locomotor activity in rats (believed to be norepinephrinergic) but that it is of comparable potency in inhibiting uptake by dopamine neurons or in eliciting stereotyped compulsive gnawing in rats, believed to be a dopaminergic-mediated syndrome.

Further suggestive evidence for levoamphetamine's dopaminergic mode of action may lie in its apparent slow attainment of efficacy compared with dextroamphetamine on psychiatrists' ratings (see figure 1). Such slow-starting efficacy in hyperkinetic children has also been reported (14) for magnesium pemoline, believed to be strongly dopaminergic (15). This slow onset of action also recalls that of the major tranquilizers, whose antipsychotic properties are suspected to be inextricably linked to their typical extrapyramidal side effects (which are probably antidopaminergic). However, no conclusions should be drawn on the basis of these scanty data, especially since the temporal pattern seen on psychiatrists' ratings does not emerge on teachers' or parents' ratings.

Supporting a dopaminergic mediation of aggression control, McKenzie(16) reported that apomorphine, believed to be a specific dopaminergic agonist, induces spontaneous aggression in male rats. Although this would seem to imply that dopaminergic drugs should exacerbate aggressive behavior, we must remember that some drugs have a paradoxical, reverse effect on some hyperkinetic children. At least, this finding supports the suspicion that dopaminergic receptors are "where the action is" for aggressive behavior.

In this light the data presented here suggest the following hypothesis for further testing: that the unsocialized-aggressive subgroup of hyperkinetic children may benefit equally from either dextroamphetamine or levoamphetamine via a dopaminergic mechanism, in contrast to the overanxious and hyperkinetic children who are helped significantly by only dextroamphetamine via a norepinephrinergic mechanism. This hypothesis is far from established, but there does seem to be enough suggestive evidence to warrant further investigations along these lines.

\section{References}

1. Fish B: The "one child, one drug" myth of stimulants in hyperkinesis. Arch Gen Psychiatry 25:193-203, 1971

2. Wender P: Minimal Brain Dysfunction in Children. New York, John Wiley \& Sons, 1971

3. Bradley C: Benzedrine and Dexedrine in the treatment of children's behavioral disorders. Pediatrics 5:24-36, 1950

4. Laufer M, Denhoff E: Hyperkinetic behavior syndrome in children. J Pediatr 50:463-473, 1957

5. Barcai A: Predicting the response of children with learning disabilities and behavior problems to dextroamphetamine sulfate. Pediatrics 47:73-79, 1971

6. Yoss RE, Moyer N: The pupillogram of the hyperkinetic child and the underachiever. Read at the Seventh Colloquium on the Pupil, Mayo Clinic, Rochester, Minn, May 13-15, 1971

7. Knopp W, Arnold LE, Andras R: Electronic pupillography: predicting amphetamine benefit in hyperkinetics. Read at the 125th annual meeting of the American Psychiatric Association, Dallas, Tex, May 1-5, 1972 
8. Corson SA, Corson E O'L, Kirilcuk V, et al: Tranquilizing effects of d-amphetamine on hyperkinetic untrainable dogs. Fed Proc 30:206, 1971

9. Corson SA, Corson E O'L, Kirilcuk V, et al: Bridging the gap between psychobiology and medical practice- development of an animal model for the study of the hyperkinetic syndrome, in Psychiatric Eclecticism: A Festschrift for Ralph Patterson. Edited by Goldman H. Columbus, Ohio, Ohio State University Press, 1972, pp $131-142$

10. Corson SA, Corson E O'L: Pavlovian conditioning as a method for studying the mechanisms of action of minor tranquilizers. Neuropsychopharmacology 129:857-881, 1967

11. Arnold LE, Wender P, McCloskey K, et al: Levoamphetamine and dextroamphetamine: comparative efficacy in the hyperkinetic syndrome. Arch Gen Psychiatry 27:816-822, 1972

12. Nichamin SJ, Barahal GD: Faulty neurologic integration with perceptual disorders in children. Mich Med 67:1071-1075, 1968

13.Snyder S, Taylor K, Coyle J, et al: The role of brain dopamine in behavioral regulation and the actions of psychotropic drugs. Am JPsychiatry 127:117-125, 1970

14. Page JG: A double-blind clinical study of pemoline in MBD. Pediatrics (in press)

15. Tagliamonte A, Tagliamonte P, Perez-Cruet J, et al: Stimulation of brain dopamine turnover by Mg pemoline. J Neurochem 18:1831-1836, 1971

16. McKenzie G: Apomorphine-induced aggression in the rat. Brain Res 34:323-330, 1971

${ }^{i}$ The unsocialized-aggressive group included two brothers with a chronic history of frequent serious fighting, stealing, and lying; a boy who had been expelled from two schools for misbehavior and had set a $\$ 1,200$ fire; and two other boys who were repeatedly suspended from school for fighting and other aggressive behavior and whose mothers asked for tranquilizers for themselves by the end of the boys' second week on placebo. One of these boys had thrown furniture at other children in the classroom. Both had a tendency to blame everyone else for their trouble; this sometimes verged on paranoia. Their parents seemed less adequate than those of the other two groups.

The overanxious group was characterized by likability, subjective distress, anxiety, some insight, at least one loving, stable parent, and generally higher intelligence than the other two groups.

The classical hyperkinetic group were also characterized by likability and at least one stable, loving parent. One of the children in this group was a postmeningitic kindergartener. Another carried the only diagnosis of mental retardation (borderline) in the study. His mother was concerned about his occasional bullying of smaller children, but this was not the most prominent manifestation of his problems, and the school did not complain about it. Because of his general disorganization and inadequacy, it seemed most appropriate to place him in this group rather than in the unsocialized-aggressive group. 